\title{
A GENERALIZED VAN KAMPEN-FLORES THEOREM
}

\author{
K. S. SARKARIA
}

(Communicated by Frederick R. Cohen)

\begin{abstract}
The $n$-skeleton of a $(2 n+2)$-simplex does not embed in $\mathbf{R}^{2 n}$. This well-known result is due (independently) to van Kampen, 1932, and Flores, 1933, who proved the case $p=2$ of the following:

Theorem. Let $p$ be a prime, and let $s$ and $l$ be positive integers such that $l(p-1) \leq p(s-1)$. Then, for any continuous map $f$ from $a(p s+p-2)$ dimensional simplex into $\mathbf{R}^{l}$, there must exist $p$ points $\left\{x_{1}, \ldots, x_{p}\right\}$, lying in pairwise disjoint faces of dimensions $\leq s-1$ of this simplex, such that $f\left(x_{1}\right)=$ $\cdots=f\left(x_{p}\right)$.
\end{abstract}

\section{INTRODUCTION}

The first general examples of simplicial complexes which are not embeddable in a Euclidean space of twice their dimension were given in the early thirties. Van Kampen [11] and Flores [5] proved (independently) that there does not exist a continuous one-one map from $\sigma_{s-1}^{2 s}$, the $(s-1)$-skeleton of a $2 s$-dimensional simplex, into the Euclidean space $\mathbf{R}^{2(s-1)}$. Both van Kampen and Flores used deleted functors (though in different ways) and both proved a little more:

1.1. Van Kampen-Flores theorem. For any continuous map $f: \sigma_{s-1}^{2 s} \rightarrow \mathbf{R}^{2(s-1)}$ there exists a pair $\left(\sigma_{1}, \sigma_{2}\right)$ of disjoint simplices of $\sigma_{s-1}^{2 s}$ such that $f\left(\sigma_{1}\right) \cap f\left(\sigma_{2}\right) \neq$ $\phi$.

An equally well-known and earlier theorem of Radon [6] can also be stated in the same format:

1.2. Radon's theorem. For any linear map $f: \sigma_{l+1}^{l+1} \rightarrow \mathbf{R}^{l}$ there exists a pair $\left(\sigma_{1}, \sigma_{2}\right)$ of disjoint simplicies of $\sigma_{l+1}^{l+1}$ such that $f\left(\sigma_{1}\right) \cap f\left(\sigma_{2}\right) \neq \phi$.

Arguments similar to those given by either van Kampen [11] or Flores [5] can be used to see that the above statement is in fact true for all continuous maps $f: \sigma_{l+1}^{l+1} \rightarrow \mathbf{R}^{l}$. This generalization of Radon's theorem was however pointed out only much later by Bajmóczy and Bárány [1].

Received by the editors August 8, 1988 .

1980 Mathematics Subject Classification (1985 Revision). Primary 57Q35, 52A37. 
The deleted functor methods used by van Kampen and Flores have been ameliorated in many different ways, and have been applied to get many interesting topological and combinatorial results, see, e.g. $[13,12,7,8,9]$, etc. The object of this note is to use the techniques of [8], where we used deleted joins to prove a generalized Erdös-Kneser conjecture, to establish the following very general theorem of the Radon-van Kampen-Flores type. Here $n, s, j, p$, and $l$ are integers with $1 \leq s \leq n+1,2 \leq j \leq p, 0 \leq l$, and $p$ is a prime, and a $p$-tuple of sets is called $j$-wise disjoint if the intersection of any $j$ of the sets is empty.

1.3. Theorem. Let either (a) $n=s-1$ and $(n+1)(j-1)>(l+1)(p-1)$, or (b) $n>s-1$, and $(m+1)(p-1)+p s \geq(n+1)(j-1)>(l+m+2)(p-1)$ for some non-negative integer $m$. Then, for any continuous map $f: \sigma_{s-1}^{n} \rightarrow \mathbf{R}^{l}$, there exists a $j$-wise disjoint p-tuple $\left(\sigma_{1}, \ldots, \sigma_{p}\right)$ of simplices of $\sigma_{s-1}^{n}$ such that $f\left(\sigma_{1}\right) \cap \cdots \cap f\left(\sigma_{p}\right) \neq \varnothing$.

When $n=s-1=(l+1)(p-1), p$ any prime, and $j=2$ this gives the

1.4. Bárány-Shlosman-Szücs theorem. For any continuous map $f: \sigma_{(l+1)(p-1)}^{(l+1)(p-1)}$ $\rightarrow \mathbf{R}^{l}$ there exists a pairwise dișioint p-tuple $\left(\sigma_{1}, \ldots, \sigma_{p}\right)$ of simplices of $\sigma_{(l+1)(p-1)}^{(l+1)}$ such that $f\left(\sigma_{1}\right) \cap \cdots \cap f\left(\sigma_{p}\right) \neq \varnothing$.

This generalization [2] of Radon's theorem remains ture for any $p$ provided one assumes that $f$ is linear: this was established earlier by Tverberg [10].

Note that the conclusion of 1.4 is stronger than saying that $f$ admits a $p$ fold multiple point, $f\left(x_{1}\right)=\cdots=f\left(x_{p}\right)$. If the complementary dimension of the diagonal of $\left(\mathbf{R}^{l}\right)^{p}$, i.e. $l(p-1)$, is more than $p$ times the dimension of a simplicial complex $K$, then any general position linear map $f: K \rightarrow \mathbf{R}^{l}$ will have no such multiple points. On the other hand by putting $n=p s+p-2, p$ any prime, $j=2$, and $m=0$, Theorem 1.3 yields the following:

1.5. Generalized van Kampen-Flores theorem. For any continuous map $f$ : $\sigma_{s-1}^{p s+p-2} \rightarrow \mathbf{R}^{l}, l(p-1) \leq p(s-1)$, there exists a pairwise disjoint $p$-tuple $\left(\sigma_{1}, \ldots, \sigma_{p}\right)$ of simplices of $\sigma_{s-1}^{p s+p-2}$ such that $f\left(\sigma_{1}\right) \cap \cdots \cap f\left(\sigma_{p}\right) \neq \varnothing$.

We note that in this result the number $p s+p-2$ is best possible. Thus, e.g. 1.5 tells us that a continuous map from the 1000-dimensional complex $\sigma_{1000}^{5008}$ to $\mathbf{R}^{1250}$ must have a 5-tuple point. But, by sending 4 vertices to each of the 1251 vertices, and the barycenter, of a 1250-dimensional simplex contained in $\mathbf{R}^{1250}$ one gets a linear map $\sigma_{1000}^{5007} \rightarrow \mathbf{R}^{1250}$ without any 5-tuple points. Similarly one has linear maps $f: \sigma_{s-1}^{p s+p-3} \rightarrow \mathbf{R}^{l}$ without $p$-tuple points whenever $l(p-1)=$ $p(s-1)$.

One lacuna in all these results is that $p$ has to be prime. However, note that the cases $j>2$ of Theorem 1.3 also give us information about the existence of $q$-fold multiple points of $f$ for nonprimes $q$ : Let us call a $p$-tuple of points $\left(x_{1}, \ldots, x_{p}\right) j$-wise distinct if no $j$ of them are the same. Note that there are 
at least $q=] p /(j-1)[$ pairwise distinct entries in such a $p$-tuple. (Here ]t denotes the smallest integer $\geq t$.) The conclusion of Theorem 1.3 is stronger than saying that $f$ must image some $j$-wise distinct $p$-tuple of points to the same point of $\mathbf{R}^{l}$, and this last is equivalent to saying that $f$ must have a $q$-fold multiple point.

\section{DELETED PRODUCTS}

As in [8] each ordered $p$-tuple of simplices of a simplicial complex $K$ will be considered to be a simplex of the $p$-fold join $K^{(p)}=K \cdot K \cdots \cdots K$ (the join of $p$ disjoint copies of $K$ ), and the deleted join $K_{(j)}^{(p)}$ is a subcomplex of $K^{(p)}$ formed by all $j$-wise disjoint ordered $p$-tuples of simplices of $K$.

Definition 2.1. Likewise, for any topological space $E$, the deleted product $E_{j}^{p}$ is the subspace of the $p$-fold product $E^{p}=E \times E \times \cdots \times E$ formed by all $j$-wise distinct ordered $p$-tuples of points of $E$. Thus we have an increasing sequence of spaces $\phi=E_{1}^{p} \subseteq E_{2}^{p} \subseteq \cdots \subseteq E_{p}^{p} \subseteq E_{p+1}^{p}=E^{p} . E_{2}^{p}$ and $E_{p}^{p}$ are also denoted by $E_{\star}^{p}$ and $E_{*}^{p}$ : these are called, respectively, the $p$ th product configuration and the $p$ th deleted product of $E$. The group $\Sigma_{p}$ of all permutations of $\{1,2, \cdots, p\}$, and therefore the subgroup $Z_{p}$ generated by the cyclic permutation $(2,3, \ldots, p, 1)$, acts on these spaces in the obvious way: $\pi\left(x_{1}, \ldots, x_{p}\right)=\left(x_{\pi(1)}, \ldots, \pi_{\pi(p)}\right) \forall \pi \in \Sigma_{p}$. Note that, for $j \leq p$, the $Z_{p}$-action on $E_{j}^{p}$ is fixed-point-free; further, if $p$ is prime, then this action is free.

2.2. The $p$ th deleted product of the Euclidean space $\mathbf{R}^{l}$ is the complement in $\mathbf{R}^{l p} \cong\left(\mathbf{R}^{l}\right)^{p}$ of the $l$-dimensional vector subspace given by $x_{1}=x_{2}=\cdots=x_{p}$. By projecting orthogonally on the perpendicular $(l p-l)$-dimensional vector subspace, and normalizing, we see that $\left(\mathbf{R}^{l}\right)_{*}^{p}$ has the $Z_{p}$-homotopy type of an (lp-l-1)-dimensional sphere. (More generally, $\left(\mathbf{R}^{l}\right)_{k}^{p}, k \leq p$, is the complement in $\mathbf{R}^{l p}$ of $\left(\begin{array}{l}p \\ k\end{array}\right), l(p-k+1)$-dimensional vector subspaces given by $x_{i_{1}}=x_{i_{2}}=\cdots=x_{i_{k}}, 1 \leq i_{1}<\cdots<i_{k} \leq p$, and one can check that this complement has the $Z_{p}$-homotopy type of a bouquet of $(l k-l-1)$-spheres.) We will also use the fact (cf. [8]) that for $\sigma_{n}^{n}$, the simplicial complex formed by all the faces of an $n$-simplex, the deleted join $\left(\sigma_{n}^{n}\right)_{(j)}^{(p)}$ has the $Z_{p}$-homotopy type of a bouquet of $((n+1)(j-1)-1)$-dimensional spheres: This follows because $\left(\sigma_{n}^{n}\right)_{(j)}^{(p)} \cong\left(\left(\sigma_{0}^{0}\right)^{(n+1)}\right)_{(j)}^{(p)} \cong\left(\left(\sigma_{0}^{0}\right)_{(j)}^{(p)}\right)^{(n+1)}$ using $(A \cdot B)_{(j)}^{(p)} \cong A_{(j)}^{(p)} \cdot B_{(j)}^{(p)}$, and so $\cong\left(\sigma_{j-2}^{p-1}\right)^{(n+1)}$. Here $\sigma_{t}^{i}$ denotes the $t$-skeleton of an $i$-simplex.

2.3. To relate the notions of joins and products we make the following conventions:

Let $\tau^{p-1}$ denote the $(p-1)$-simplex whose vertices are the canonical basis vectors $(0, \ldots, 0,1,0, \ldots, 0)$ of $\mathbf{R}^{p}$. The $p$-fold join of spaces, $E^{(p)}=$ $E \cdot E \cdots E$ (the join of $p$ disjoint copies of $E$ ) has as its points all formal 
linear combinations $t \cdot x=t_{1} x_{1}+\cdots+t_{p} x_{p}, t \in\left|\tau_{p-1}^{p-1}\right|, x \in E^{p}$, with $t \cdot x$ identified with $t^{\prime} \cdot x^{\prime}$ whenever $t=t^{\prime}$ and $x_{i}=x_{i}^{\prime} \forall i$ s.t. $t_{i}=t_{i}^{\prime} \neq 0$. The group $\Sigma_{p}$ acts on $E^{(p)}$ via $\pi\left(t_{1} x_{1}+\cdots+t_{p} x_{p}\right)=t_{\pi(1)} x_{\pi(1)}+\cdots+t_{\pi(p)} x_{\pi(p)}$. We identify $E^{p}$ with the subspace of $E^{(p)}$ consisting of points of the type $(1 / p) \cdot x=(1 / p) x_{1}+\cdots+(1 / p) x_{p}$. On the other hand $K^{p}=K \times K \times \cdots \times K$ (resp. $K_{j}^{p}$ ) is the cell complex whose cells correspond to (resp. $j$-wise disjoint) ordered $p$-tuples of nonempty simplices of $K$. Alternatively one can consider $K^{p}$ as a subposet of $K^{(p)}$ under $\subseteq$. Under barycentric subdivision this subposet yields a subcomplex $\left(K^{p}\right)^{\prime}$ of $\left(K^{(p)}\right)^{\prime}$ and, if $E=|K|$, hen $E^{(p)}$ is covered by $K^{(p)}$ and the subspace $E^{p}$ is covered by the subcomplex $\left(K^{p}\right)^{\prime}$ of $\left(K^{(p)}\right)^{\prime}$. The complement $K^{(p)}-K^{p}$, a subcomplex of $K^{(p)}$, will be denoted by $K_{0}^{(p)}$ (: its elements are ordered $p$-tuples of simplices of $K$, with at least one member of the $p$-tuple empty). The subspace of $E^{(p)}$ covered by $K_{0}^{(p)}$ will be denoted by $E_{0}^{(p)}$. Note that $E_{0}^{(p)}$ consists of all points of the type $t \cdot x$, with $t$ lying in the $(p-2)$-sphere $\left|\tau_{p-2}^{p-1}\right|$. The group $\Sigma_{p}$ acts on this sphere via $\pi\left(t_{1}, \ldots, t_{p}\right)=$ $\left(t_{\pi(1)}, \ldots, \tau_{\pi(p)}\right)$.

\section{Multiple points}

The proof of 1.3 will be based on

Lemma 3.1. Let $p$ be a prime number, and $K$ a simplicial complex. If there exists a continuous map $f$ from $|K|$ into $\mathbf{R}^{l}$, with $f\left(\sigma_{1}\right) \cap \cdots \cap f\left(\sigma_{p}\right)=\phi \forall$ $j$-wise disjoint $p$-tuples of simplices of $K$, then there must also exist a continuous map $F$ from $\left|K_{(j)}^{(p)}\right|$ to the sphere $S^{(l+1)(p-1)-1}$, which commutes with some free actions of the group $Z_{p}$.

This is an easy consequence of the functorial nature of deleted products: Any map $E \stackrel{f}{\longrightarrow} Y$ taking $j$-wise distinct $p$-tuples to $k$-wise distinct $p$-tuples induces the $Z_{p}$-map

$$
f^{p}: E_{j}^{p} \rightarrow Y_{k}^{p},
$$

where $f^{p}\left(x_{1}, \ldots, x_{p}\right)=\left(f\left(x_{1}\right), \ldots, f\left(x_{p}\right)\right)$. If $E=|K|$, then with the conventions made in $2.3 E_{j}^{p}$ is bigger than $\left|K_{j}^{p}\right|=\left|\left(K_{j}^{p}\right)^{\prime}\right|$. The latter is the subspace of $j$-wise distinct $p$-tuples of points which are contained in $j$-wise disjoint $p$-tuples of simplices, and so one has a map $f^{p}:\left|K_{j}^{p}\right| \rightarrow Y_{k}^{p}$ even under the weaker hypothesis that all such $p$-tuples are mapped by $f$ to $k$-wise distinct $p$-tuples of $Y .\left|K_{(j)}^{(p)}\right| \subset E_{0}^{(p)} \cdot\left|K_{j}^{p}\right|$, and one has the $Z_{p}$-map $\lambda: E_{0}^{(p)} \rightarrow\left|\tau_{p-2}^{p-1}\right|$ given by $\lambda(t \cdot x)=t$. Thus we get the $Z_{p}$-map

$$
\lambda \cdot f^{p}:\left|K_{(j)}^{(p)}\right| \rightarrow\left|\tau_{p-2}^{p-1}\right| \cdot Y_{k}^{p} .
$$

The result follows because 2.2 tells us that, when $Y=\mathbf{R}^{l}$ and $k=p$, the right side of (3.3) has the $Z_{p}$-homotopy type of an $S^{(l+1)(p-1)-1}$. 
3.4. Proof of Theorem 1.3. If some $f:\left|\sigma_{s-1}^{n}\right| \rightarrow \mathbf{R}^{l}$ does not have the stated property, then the above lemma provides us with a $Z_{p}$-map $F:\left|\left(\sigma_{s-1}^{n}\right)_{(j)}^{(p)}\right| \rightarrow$ $S^{(l+1)(p-1)-1}$. In case (a) no such $F$ can exist because 2.2 tells us that connectivity of the domain is $\geq$ the dimension of the range, and the Borsuk-Ulam result of Dold [4], p. 68, applies. Even in case (b), instead of ruling out the existence of such an $F$ by means of a direct examination of the connectivity of $\left(\sigma_{s-1}^{n}\right)_{(j)}^{(p)}$ we will make do with the same readily available information 2.2 regarding $\left(\sigma_{n}^{n}\right)_{(j)}^{(p)}$.

We note that the barycentrically subdivided complex $\left(\left(\sigma_{n}^{n}\right)_{(j)}^{(p)}\right)^{\prime}$ is contained in the join of the two "complementary" subcomplexes, $\left(\left(\sigma_{s-1}^{n}\right)_{(j)}^{(p)}\right)^{\prime}$ and $L=$ $\left(\left(\sigma_{n}^{n}\right)_{(j)}^{(p)}-\left(\sigma_{s-1}^{n}\right)_{(j)}^{(p)}\right)^{\prime}$. Hence, if we could find a $Z_{p}$-map $G:|L| \rightarrow S^{(m+1)(p-1)-1}$, then the join of maps $F \cdot G$ would provide us with a $Z_{p}$-map $\left|\left(\left(\sigma_{n}^{n}\right)_{(j)}^{(p)}\right)^{\prime}\right| \rightarrow$ $S^{(l+m+2)(p-1)-1}$. And, no such $Z_{p}$-map could exist, because, under the given condition $(n+1)(j-1)>(l+m+2)(p-1)$, the domain has connectivity $\geq$ the dimension of the range.

The map $G$ can be defined (cf. [8]) as follows. Let $\sigma^{n}=\{1,2, \ldots, n+1\}$, $\theta^{m}=\{1,2, \ldots, m+1\}$, and assign to each $s$-face $\xi^{s} \subset \sigma^{n}$, a color $c\left(\xi^{s}\right) \in \theta^{m}$ thus: if $\xi^{s}$ has a vertex

$$
\left.\leq m \frac{p-1}{j-1}, \quad \text { then } c\left(\xi^{s}\right)=\right] \frac{\left(\text { first vertex of } \xi^{s}\right)(j-1)}{p-1}[,
$$

otherwise $c\left(\xi^{s}\right)=m+1$. Using the given condition $(m+1)(p-1)+p s \geq$ $(n+1)(j-1)$ it is easily checked that one cannot have $c\left(\xi_{1}^{s}\right)=\cdots=c\left(\xi_{p}^{s}\right)$ for any $j$-wise disjoint $p$-tuple $\left(\xi_{1}^{s}, \cdots, \xi_{p}^{s}\right)$ of $s$-faces of $\sigma^{n}$. (Because $\left|\xi_{1}^{s} \cup \cdots \cup \xi_{p}^{s}\right| \geq p(s+1) /(j-1)$ while length $(m(p-1) /(j-1), n+1]<$ $p(s+1) /(j-1)$, all $\xi_{i}^{s} s$ could not have been colored by $m+1$. Also the total number of vertices, occurring first in some $\xi_{i}^{s}$, is $\geq p /(j-1)$; so, if all first vertices were $\leq m(p-1) /(j-1)$, then largest color-smallest color $\geq](p /(j-1)-1)(j-1) /(p-1)[=1$. $)$ More generally, to any face $\alpha^{t} \subset \sigma^{n}$, assign the set $\bar{c}\left(\alpha^{t}\right) \subset \theta^{m}$ of colors assigned to $s$-faces of $\sigma^{n}$ contained in $\alpha^{t}$ (so $\bar{c}\left(\alpha^{t}\right)=\varnothing$ iff $\left.t<s\right)$. Now we define a $Z_{p}$-monotone function $C:\left(\sigma_{n}^{n}\right)_{(j)}^{(p)} \rightarrow$ $\left(\theta_{m}^{m}\right)_{(p)}^{(p)}$ by $C\left(\alpha_{1}, \ldots, \alpha_{p}\right)=\left(\bar{c}\left(\alpha_{1}\right), \ldots, \bar{c}\left(\alpha_{p}\right)\right)$. Note that $C\left(\alpha_{1}, \ldots, \alpha_{p}\right)$ is empty iff $\left(\alpha_{1}, \ldots, \alpha_{p}\right)$ belongs to the subcomplex $\left(\sigma_{s-1}^{n}\right)_{(j)}^{(p)}$ of $\left(\sigma_{n}^{n}\right)_{(j)}^{(p)}$. Thus the derived map of $C$ provides us with the requisite $G=\left|C^{\prime}\right|:|L| \rightarrow\left|\left(\theta_{m}^{m}\right)_{(p)}^{(p)}\right| \simeq$ $S^{(m+1)(p-1)-1}$. Q.E.D.

3.5. Remarks. (a) Van Kampen [11] and Flores [5] also point out that the complexes obtained by taking joins of complexes of type $\sigma_{s-1}^{2 s}$ retain the property of being nonembeddable in twice-dimensional Euclidean space. Analogously if the hypotheses of Theorem 1.3 are satisfied by $\left(j, p, n_{1}, s_{1}, l_{1}\right)$ and $\left(j, p, n_{2}, s_{2}, l_{2}\right)$, then the conclusion of Theorem 1.3 is valid for any contin- 
uous map $f: \sigma_{s_{1}-1}^{n_{1}} \cdot \sigma_{s_{2}-1}^{n_{2}} \rightarrow \mathbf{R}^{l_{1}+l_{2}+2}$. To see this proceed as in 3.4 to construct a $Z_{p}$-monotone function $C_{1} \cdot C_{2}:\left(\sigma_{n=n_{2}+1}^{n_{1}+n_{2}+1}\right)_{(j)}^{(p)} \rightarrow\left(\theta_{m_{1}+m_{2}+1}^{m_{1}+m_{2}+1}\right)_{(p)}^{(p)}$ and note that this function is supported on simplices not contained in the subcomplex $\left(\sigma_{s_{1}-1}^{n_{1}} \cdot \sigma_{s_{2}-1}^{n_{2}}\right)_{(j)}^{(p)}$. (b) Another generalization of Theorem 1.3 yields sufficient conditions ensuring that every continuous map $f: \sigma_{s-1}^{n} \rightarrow \mathbf{R}^{l}$ has a $j$-wise disjoint $p$-tuple $\left(\sigma_{1}, \ldots, \sigma_{p}\right)$ of simplices of $\sigma_{s-1}^{n}$ for which $\left(f\left(\sigma_{1}\right), \ldots, f\left(\sigma_{p}\right)\right)$ is a $k$-disjoint $p$-tuple of subsets of $\mathbf{R}^{l}$. This time one uses the fact that $\left(\mathbf{R}^{l}\right)_{(k)}^{(p)}$ is a bouquet of $(l+1)(k-1)-1$-spheres, and the (easy) "if" part of the following "generalized Erdös-Kneser Conjecture": There is a coloring $c$ of the s-faces of $\sigma^{n}$ by the vertices of $\theta^{m}$, with all $j$-disjoint tuples $\left(\xi_{1}^{s}, \ldots, \xi_{p}^{s}\right)$ going to $k$ distinct tuples $\left(c\left(\xi_{1}^{s}\right), \ldots, c\left(\xi_{p}^{s}\right)\right)$ if and only if $(m+1)(k-1)+p s \geq(n+1)(j-1)$. The "only if" part can be established by means of a slight generalization of the argument given in [8].

(c) For the classical van Kampen-Flores theorem 1.1, our proof, 3.4, is closely related to that of Flores [5]. Both proofs use the classical Borsuk-Ulam theorem [3]. In addition, Flores used the interesting fact that $\left(\sigma_{s-1}^{2 s}\right)_{*}^{(2)}$ is $Z_{2}$ homeomorphic to a $(2 s-1)$-sphere. On the other hand, we use the more obvious fact that $\left(\sigma_{2 s}^{2 s}\right)_{*}^{(2)}$ is a $2 s$-sphere, and that, there is a coloring $c$ of the $s$-faces of $\sigma_{2 s}^{2 s}$ with just one color since no two $s$-faces can be disjoint.

(d) Van Kampen's proof [11] was self-contained and did not depend on the contemporaneous work of Borsuk [3] (in fact an argument similar to, and easier than, that given by van Kampen, also gives a simple proof of the BorsukUlam theorem): To each general position map $f$ from $K$ to an oriented twicedimensional Euclidean space, associate the integral cocycle $o_{f}$ of $K_{*}^{2} / Z_{2}$ which counts (with sign) the number of intersections, under $f$, of any pair of disjointoriented simplices of $\mathrm{K}$. Van Kampen checked that a deformation of $f$ changes $\mathfrak{o}_{f}$ only by a coboundary. Thus the value $\langle\mathfrak{o}, z\rangle$ of $\mathfrak{o}_{f}$ on a cycle $z$ of $K_{*}^{2} / Z_{2}$ is independent of $f$. The nonembeddability of $\sigma_{s-1}^{2 s}$ in $\mathbf{R}^{2(s-1)}$ follows by noting that there is a general position map $f: \sigma_{s-1}^{2 s} \rightarrow \mathbf{R}^{2(s-1)}$ with just one double point and so $\langle\mathfrak{o}, z\rangle=1 \bmod 2$ for the $\bmod 2$ cycle $z$ formed by all topdimensional cells of $\left(\sigma_{s-1}^{2 s}\right)_{*}^{2} / Z_{2}$. We note that the linear-homotopy-theoretical argument of Tverberg [10] is analogous to that of van Kampen [11]. We will show elsewhere that van Kampen type arguments can be used to establish a number of interesting results besides those of Dold [4], and of this paper. A more leisurely and extensive account of the numerous combinatorial and topological applications of deleted functors will be given in [9].

\section{REFERENCES}

1. E. G. Bajmóczy and I. Bárány, On a common generalization of Borsuk's and Radon's theorem, Acta Math. Acad. Sci. Hungar. 34 (1979), 347-350.

2. I. Bárány, S. B. Shlosman and A. Szücs, On a topological generalization of a theorem of Tverberg, J. London Math. Soc. (2) 23 (1981), 158-164. 
3. K. Borsuk, Drei Sätze über die n-dimensionale euklidische Sphäre, Fund. Math. 20 (1933), 177-190.

4. A. Dold, Simple proofs of some Borsuk-Ulam results, Contemp. Math., vol. 19, Amer. Math. Soc., 1983, pp. 65-69.

5. A. Flores, Über n-dimensionale Komplexe die im $R_{2 n+1}$ absolut selbstverschlungen sind, Ergeb. Math. Kolloq. 6 (1933/34), 4-7.

6. J. Radon, Mengen konvexer Körper, die einen gemeinsamen Punkt enthalten, Math. Ann. 83 (1921), 113-115.

7. K. S. Sarkaria, Kneser colorings of polyhedra, Illinois J. Math. (to appear).

8. __ A generalized Kneser conjecture, J. Combin. Theory Ser B (to appear).

9. $ـ$ Van Kampen obstructions, in preparation.

10. H. Tverberg, A generalization of Radon's theorem, J. London Math. Soc. 41 (1966), 123128.

11. E. R. van Kampen, Komplexe in euklidischen Räumen, Abh. Math. Sem. Hamburg 9 (1932), 72-78; Berichtigung dazu, ibid (1932), 152-153.

12. C. Weber, Plongements de polyèdres dans le domaine métastable, Comment. Math. Helv. 42 (1967), 1-27.

13. W.-T. Wu, $A$ theory of imbedding, immersion, and isotopy of polytopes in a Euclidean space, Science Press, Peking, 1965.

9166 Barrick Street, Apt. 203, Fairfax, Virginia 22031

Current address: Department of Mathematics, Panjab University, Chandigarh 160014, India 